\title{
Task experience influences coordinative structures and performance variables in learning a slalom ski-simulator task
}

\section{A. Dutt-Mazumder ${ }^{1}$ (D) ～K. M. Newell ${ }^{2}$}

${ }^{1}$ Department of Radiology, University of Michigan, Ann Arbor, MI, USA

${ }^{2}$ Department of Kinesiology, University of Georgia, Athens, GA, USA

\section{Correspondence}

Aviroop Dutt-Mazumder, Department of Radiology, University of Michigan, Ann Arbor, MI, USA.

Email: daviroop@umich.edu
The experiment investigated the progressions of the qualitative and quantitative changes in the movement dynamics of learning the ski-simulator as a function of prior-related task experience. The focus was the differential timescales of change in the candidate collective variable, neuromuscular synergies, joint motions, and task outcome as a function of learning over 7 days of practice. Half of the novice participants revealed in day 1 a transition of in-phase to anti-phase coupling of center of mass (CoM)-platform motion whereas the remaining novices and experienced group all produced on the first trial an anti-phase CoM-platform coupling. The experienced group also had initially greater amplitude and velocity of platform motion-a performance advantage over the novice group that was reduced but not eliminated with 7 days of practice. The novice participants who had an in-phase CoM-platform coupling on the initial trials of day 1 also showed the most restricted platform motion in those trials. Prior-related practice experience differentially influenced the learning of the task as evidenced by both the qualitative organization and the quantitative motion properties of the individual degrees of freedom ( $d o f)$ to meet the task demands. The findings provide further evidence to the proposition that CoM-platform coupling is a candidate collective variable in the ski-simulator task that provides organization and boundary conditions to the motions of the individual joint $d o f$ and their couplings.

\section{K E Y W O R D S}

multi-joint coordination, skill acquisition, slalom ski-simulator, synergies

\section{1 | INTRODUCTION}

Bernstein $^{1}$ postulated that learning was in essence, the mastery of the redundant degrees of freedom (dof) in joint coordination dynamics as a function of practice. He conceptualized that a novice performer learned to coordinate and control a large number of mechanical dof while attempting to learn a novel motor task. Bernstein proposed that novices would to the degree necessary freeze out the dof (joint angles) at the initial learning stage—an hypothesis that has been experimentally supported in the motions of arm segments in pistol shooting ${ }^{2}$ and torso and leg motions in the ski-simulator task, ${ }^{3}$ although the particular order to freezing and freeing (releasing) of the joint motion appears strongly task-dependent.

In the ski-simulator dynamic balance task, however, freezing the joint angles to project the CoM of the body vertically over the platform tends to result in an in-phase (upright standing posture-like) coupling between CoM and the platform, a kinematic relation that limits the range of lateral motion of the platform. Indeed, the in-phase macroscopic coordination relation is relatively unstable on the platform and cannot realize the task goal of large, fluid, and efficient platform movement (amplitude and velocity) as reflected in a skilled ski-simulator performance. Thus, the macroscopic CoMplatform coupling sets boundary conditions on the platform 
motion as determined in the task space of its amplitude and velocity. ${ }^{4,5}$

The CoM and platform relative motion reflects an emergent property of the qualitative and quantitative movement dynamics that we examine here in the progressions of learning the dynamic balance ski-simulator task as a function of priorrelated task experience. A primary question was whether the macroscopic phase transition of the CoM-platform motion occurs only after considerable practice and a change in the intrinsic dynamics as found in learning the roller ball task ${ }^{6}$ or whether it occurs following limited practice as a consequence of familiarization and "getting the idea" of the task. ${ }^{7-9}$ The latter concept of "getting the idea" has been long held to occur in the early stage of motor skill learning but typically it has not been studied directly in terms of qualitative movement kinematics. Qualitative is used in the context of a different nominal macroscopic movement pattern such as in-phase and anti-phase of CoM to platform.

Here, we examine this feature of learning in terms of the presence and/or change of the macroscopic movement dynamics as a function of learning. The proposition to be investigated is that a qualitative variable such as CoM-platform in-phase relative motion constrains the organization of the joint and synergy motions, and thus, the learning of the skisimulator task requires an anti-phase pattern to its organization for successful performance. Getting this macroscopic organization in the movement dynamics can be viewed as the dynamical essence of "getting the idea" of the task. Moreover, a rapid timescale of change of the CoM-platform phase relation would support the interpretation that both the in-phase and the anti-phase CoM-platform dynamics are relatively stable states of the dynamics as in the HKB model for bimanual control. ${ }^{10}$

The proposition that CoM-platform coupling may be a candidate collective variable in the whole-body ski-simulator task has experimental rationale. That CoM is a property of the macroscopic variable follows from it being a spatial point where the body mass is distributed equally in all directions thus making it fundamental to postural balance tasks. ${ }^{4}$ It is also consistent with the evidence for $\mathrm{CoM}-\mathrm{CoP}$ as the candidate collective variable in experimental investigations of standing posture ${ }^{5}$ and an externally driven dynamic platform balance protocol. ${ }^{11}$ An important consequence of the several joint space degrees of freedom in whole-body dynamic balance tasks is that they allow in principle, unlike the restricted 2 dof bimanual coordination task, ${ }^{10}$ the independent consideration of the motion of a macroscopic variable from that of particular neuromuscular joint synergies (couplings) and individual joints. This broader range of variables in the task dynamics allows a test of the notion of reciprocal causality of the organization of the macroscopic variable with the individual joint motion and synergies. ${ }^{11}$ Thus, the ski-simulator task with many joint dof motions involved affords a rich context for movement dynamics and the study of the rates of change of the motions of candidate collective variable, neuromuscular synergies, individual joints, and platform as a function of prior practice experience, and days of practice in the ski task.

In coordination dynamics, ${ }^{12}$ the changes in movement dynamics with learning are embedded within the formation of the collective variable through reciprocal causality with the individual dof joint motions and couplings of varying relations, strengths, and timescales between the multiple dof-adjoining phase relations in joint space. ${ }^{13-16}$ Indeed, Bernstein ${ }^{1}$ proposed that the three stages of learning a novel skill involve freezing out mechanical dof of joints, gradually releasing the constrained $d o f$, and finally exploiting (taking advantage of) the reactive forces of the motions of the dof to produce economical and efficient movement as a function of practice. This pathway of change in motor learning eventually leads to fluent, rhythmical, and large amplitude movement in the ski-simulator task, ${ }^{3,17}$ including potentially an anti-phase coupling of CoM-platform motion that is required (albeit implicitly) to realize the task demands. ${ }^{14}$

In this study, we examined the hypothesis that the timescales of change in forming the respective couplings would be different for the macroscopic variable (CoM-platform motion) than the neuromuscular synergy variables as a function of the control parameter, that is, the increasing platform amplitude and velocity motion that emerges with practice. ${ }^{13,14} \mathrm{It}$ was anticipated that if the anti-phase collective variable was not produced in the initial trials of practice it would emerge from a transition at some point in practice to an anti-phase pattern. In contrast, the synergy variables would reflect transient and faster timescale change with different relative phase values than the candidate collective variable over the progression of practice.

Furthermore, it was hypothesized that the participants experienced in task-related activities would produce an earlier and more distinct anti-phase coupling than the novice participants even though they had not practiced the specific lateral motion ski-simulator task studied here. This is consistent with the proposition that the intrinsic dynamics provide boundary conditions to the organization movement of coordination and control and the change of it in learning, retention, and transfer. ${ }^{11}$ The time of acquisition of a new bimanual relative phase has been shown to be dependent on the prior experience of the learners, ${ }^{18,19}$ including in the context of sports skills. $^{20-22}$

In general, it was expected that the rate of freeing the individual joint dof across practice days would be different as a function of prior practice and task experience and that this would be influenced by the phase relation of the macroscopic CoM-platform motion. The novice group would initially produce a more progressive rate of increment of lower limb joint angle range (freeing $d o f$ ) than the experienced group because initially in practice they would have a more restricted 
platform amplitude through reduced ankle, knee, and hip joint motion. And, to realize this change, the novice group would produce a higher variability of lower limb joint angle motion as a function of practice (exploiting dof) given that they had more change in platform motion to realize the task goal from their limited initial posture and movement conditions.

The rates of change of the motion of the candidate collective variable, synergies, individual joints, and platform would be different as a function of prior practice experience and days of practice in the ski task. The qualitative and quantitative changes in the movement dynamics would show properties of both the continuity and the discontinuity of motor skill learning ${ }^{23-26}$ that depend on prior practice experience.

\section{$2 \mid$ METHODS}

\section{1 | Participants}

Twelve healthy female participants ( $23 \pm 5$ years) were recruited according to an experimental protocol approved by The Pennsylvania State University Institutional Review Board. The participants consisted of two groups-novice and experienced skiers. The novice group had no previous experience of dynamic balancing tasks such as surfing, skiing, rollerblading, and snowboarding, whereas the experienced group consisted of experienced alpine skiers ( $>5$ years of skiing) from a local ski club team. None of the participants from either group had any previous practice experience on the ski-simulator task studied here. Their average height was $164.4 \pm 6.2 \mathrm{~cm}$, and their average mass was $53.2 \pm 4.3 \mathrm{~kg}$. There was no average group difference in height/mass. All participants self-reported no apparent neurological disorders or musculo-skeletal injuries that could negatively influence postural control.

\section{2 | Apparatus}

The ski-simulator (Skier's Edge, Utah) was the experimental apparatus that is a movable wheeled platform comprising of two codependent footplates. ${ }^{3}$ The elastic band fitted underneath the footplates facilitates lateral oscillations. A six-camera 3-D motion analysis system (QTM, Sweden) was positioned with the cameras equally distant from each other around $360^{\circ}$ of the participant to encompass the calibrated space of the test area and record the motion of passive markers that were attached to the anatomical joints of the experimental participants. The data were sampled at $100 \mathrm{~Hz}$ and were digitally low-pass filtered with a second-order Butterworth filter and a cut-off frequency of $5 \mathrm{~Hz}$. An initial assessment to determine the frequency power of the dependent variables was carried out by running an FFT. It was found that the signal power was constrained to $<2 \mathrm{~Hz}$ for all signals and subsequently analyzed consistent with an earlier study. ${ }^{13}$

\section{3 | Task and procedures}

The participant's task instruction was to make as large an amplitude and velocity side-to-side movements on the skisimulator as they could with their hands folded in the front of their torso. No additional information was provided. Each participant practiced for 140 trials spanning over 7 consecutive days. Every trial consisted of 45 seconds of practice followed by 1 minute of rest.

\section{4 | Data analysis}

\subsection{1 | Kinematic variables}

The kinematic variables were the individual joint angles (hip, knee, and ankle) calculated in the mediolateral (ML) axis that were defined based on passive markers attached to anatomical landmarks. The CoM was calculated from the 13 segment model, reconstructed from a 20 anatomical marker system-lateral side of head, shoulder (lesser tubercle of humerus), wrist (radial styloid process), elbow (lateral epicondyle of humerus), iliac (tubercle crest), hip (greater trochanter), knee (lateral femoral epicondyle), ankle (frontal talus), toe (3rd metatarsal) according to the anthropometric data of Dempster. ${ }^{27}$ Applying the weighting factors of the segmental masses, the whole-body CoM position was estimated by the weighted summation of the individual segment CoM positions. ${ }^{4}$

\subsection{2 | Cophase}

The coupled variables of CoM-platform, head-platform, hipankle, hip-knee, and knee-ankle were investigated through the cophase technique. ${ }^{28}$ For kinematic data analysis, we considered the right ipsilateral joints. Here, $0^{\circ}$ implies that the signals are coupled and in-phase, whereas anti-phase mode would be reflected by $+180^{\circ}$.

$$
\text { Cophase }(f)=\operatorname{atan} 2 \mathrm{~d}\left[-\operatorname{imag}\left(S_{a b}(f)\right) \text {, real }\left(S_{a b}(f)\right)\right]
$$

where $S_{a b}(f)$ is the cross-power spectral density of the two time series. ${ }^{29}$

The cophase characterizes the lead-lag relation of two signals as a function of frequency. For example, a $0^{\circ}$ cophase indicates an in-phase coupling that two time series simultaneously travel together. On the contrary, a $180^{\circ}$ cophase represents an anti-phase coordination that one signal has a half cycle delay to the other (eg, $-180^{\circ}$ implies that signal $y$ leads $x$ ). More precisely, if the phase difference is stable and constant over time, that is, phase locked then coherence $=1.0$ and if time difference between two signals varies from moment-to-moment then coherence $=0$. The descriptive circular statistics (mean and SD) were derived to reveal the cophase patterns for 
all articular couplings and the CoM-platform coordination qualitatively. ${ }^{30}$

\subsection{3 $\mid$ Coherence}

The coupled variables were analyzed using the Chronux toolbox. ${ }^{31}$ Coherence measures the correlation of two signals in the frequency domain where multi-taper spectral tool reduces the spectrum estimation bias by obtaining multiple independent estimates from the time series that are dependent on the sampling frequency and time series bandwidth. ${ }^{32}$ Typically, values range between 1 (perfect linear prediction between variables) and 0 (variables are linearly independent).

$$
\text { Coherence }(f)^{2}=\frac{\mid\left(\left.S_{a b}(f)\right|^{2}\right.}{S_{a}(f) \cdot S_{b}(f)}
$$

where $S_{a}(f)$ and $S_{b}(f)$ are the power spectral densities of signal $a$ and $b$, respectively.

\subsection{4 | Statistics}

A two-way mixed design repeated measures ANOVA of 7 (days) $\times 2$ (groups-novice and experienced) was carried out independently on the CoM-platform, head-platform, hip-ankle, hip-knee, and knee-ankle couplings, individual joint angle variables, and platform kinematics. The Tukey's post hoc test was used to determine the differences between all paired levels for the dependent variables. Circular statistics were used to calculate the mean and standard deviation of coherence across the trial period. ${ }^{30}$ Alpha level was set $P<.05$.

\section{3 | RESULTS}

\section{1 | Platform kinematics}

The amplitude of the lateral skiing movement for the novice group increased significantly as a function of practice days and more than the experienced group (see Figure 1), but the experienced group still produced a greater range of platform motion than the novice group on day 7 . The amplitude of platform motion of the novice group was $16.36 \pm 4.26 \mathrm{~cm}$ $(\mathrm{n}=6)$ on day 1 which increased to $37.32 \pm 1.56 \mathrm{~cm}$ on day 7. In contrast, the experienced group had a mean of $35.91 \pm 1.51 \mathrm{~cm}$ on day 1 that increased to $40.45 \pm 1.05 \mathrm{~cm}$ on day 7 .

A repeated measures ANOVA with days (7) and groups (2: novice and experienced) on platform amplitude showed a significant main effect of group, $F(1,70)=21.62, P<.05$; day, $F(6,70)=236.34, P<.05$, and a significant interaction between groups and days, $F(6,70)=10.34, P<.05$. The Tukey's post hoc test showed that all pairwise comparisons of the interaction were significant.
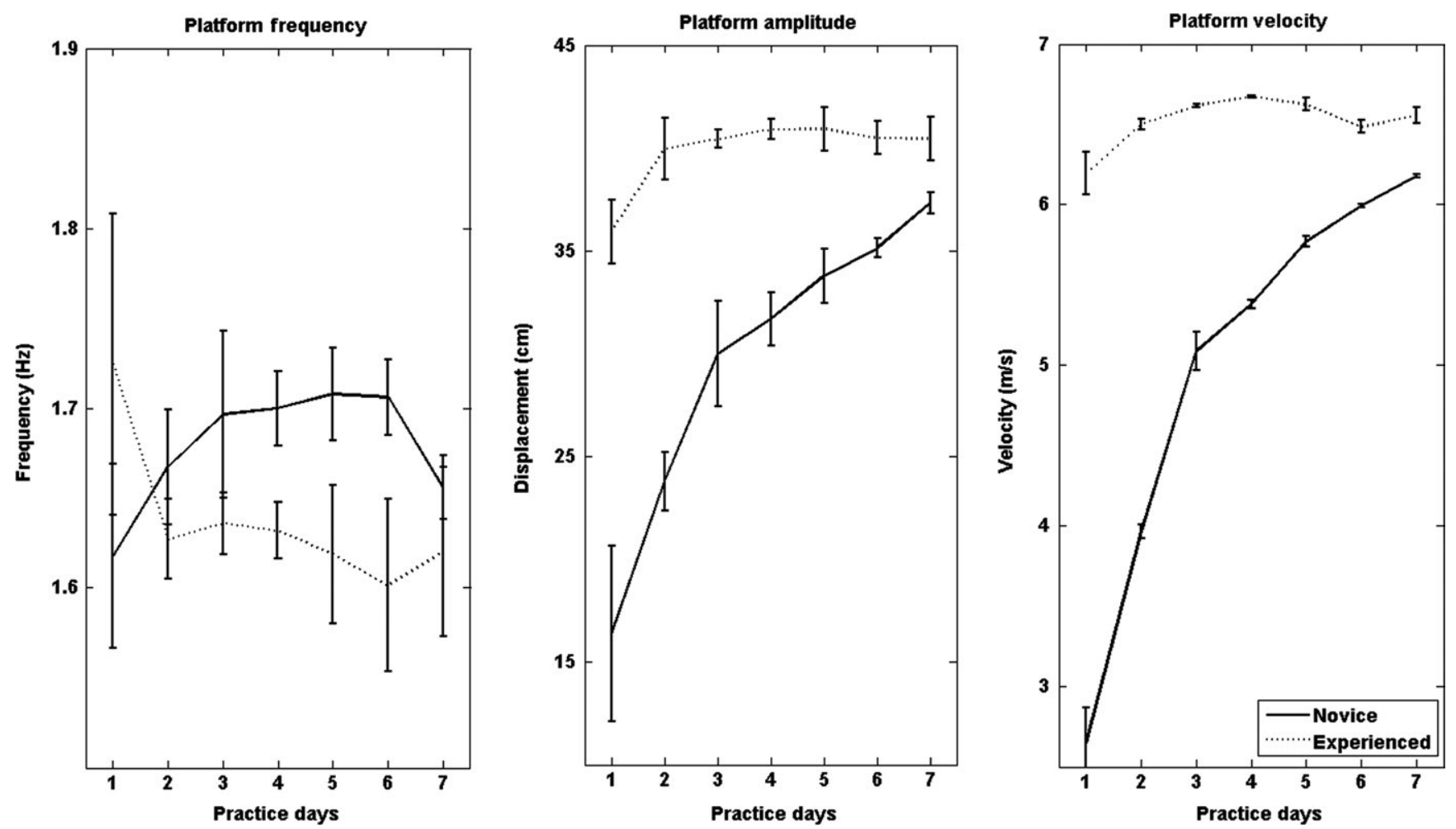

F I G U R E 1 Group mean of platform frequency, amplitude, and velocity (error bar between subjects' standard deviation) of lateral skiing movement as a function of experienced and novice groups across practice days 
A repeated measures ANOVA with days (7) and groups (2: novice and experienced) on platform frequency showed a significant main effect of group, $F(1,70)=10.07, P<.05$; day, $F(6,70)=46.37, P=.05$, and a significant interaction between groups and days, $F(6,70)=55.90, P<.05$. The Tukey's post hoc test showed that all pairwise comparisons were significant although the direction of initial group difference was reversed in days 3-7.

For platform velocity, the main effect of group was significant, $F(1,70)=489.01, P<.05$, and there was a significant interaction of group and day, $F(6,70)=201.24$ at $P<.05$. The Tukey's post hoc test showed that all pairwise comparisons were significant.

Figure 2A,B depicts example time series of platform, $\mathrm{CoM}$, and head motion early and late in practice for a novice participant who showed a phase transition of CoM-platform motion. The ML motion kinematics of $\mathrm{CoM}$, head, and platform showed an in-phase CoM-platform motion on day 1, trial 1 and subsequent performance with an anti-phase CoMplatform motion on day 7 , trial 20 , respectively. On day 1 , trial 1, the amplitude of the platform motion was highly constrained $(\sim 1 \mathrm{~cm})$ with a low CoM oscillating amplitude $(\sim 2 \mathrm{~cm})$ and a relatively large head oscillating amplitude $(\sim 7 \mathrm{~cm})$, reflecting an inverted pendulum motion, that is, larger amplitude in the distal end (eg, head) and constrained amplitude at the pivoted end (eg, platform). In contrast, for the same participant on day 7 , trial 20 , the oscillating amplitude of the platform was larger $(\sim 17 \mathrm{~cm})$, whereas the amplitude of CoM oscillated intermediately $(\sim 5 \mathrm{~cm})$, and the amplitude of head oscillation was highly conserved $(\sim 3 \mathrm{~cm})$, reflecting a hanging pendulum and an anti-phase CoM-platform mode.

Figure $2 \mathrm{C}$ reflects the platform amplitude for the first three trials on day 1 . The bold line plot represents the group mean of the novice participants, whereas the dotted line plot depicts the subset of three novice participants who did not demonstrate an anti-phase coupling of CoM and platform in the initial stage of practice. The platform amplitude appears to have a relation with the cophase values among the three novices as shown and discussed later in Figure 2D.

\section{2 | Joint angle properties}

Figure 3 and Table 1 depict the mean and $S D$ of joint angle motions - ankle, knee, and hip-for the two groups, calculated over joint angles from both sides of the body as a function of practice days. Clearly, the novices enhanced substantially their joint angle range across practice days for ankle, knee, and hip motions (see Figure 3). The novices also increased their relative standard deviation $(\mathrm{CV} \%)$ for all lower joint angles over practice days (see Table 1), that was reflected by a larger change in percentage of coefficient of variation. The experienced group essentially maintained their respective joint angle standard deviations across practice days.

A two-way repeated ANOVA of 7 (days) $\times 2$ (groupsnovice and experienced) on the knee joint angle range showed significance for group, $F(1,70)=21.07, P<.05$. A similar analysis on hip joint angle range also showed significance for the main effect of group, $F(1,70)=13.17, P<.05$. There was neither a main effect for days nor an interaction between groups and days.

A two-way repeated ANOVA of 7 (days) $\times 2$ (groupsnovice and experienced) on the knee angle showed a main effect of group, $F(1,70)=50.16, P<.05$, where the experienced group had larger joint angle motions than the novice group. Similarly, there was a main effect of group for the hip
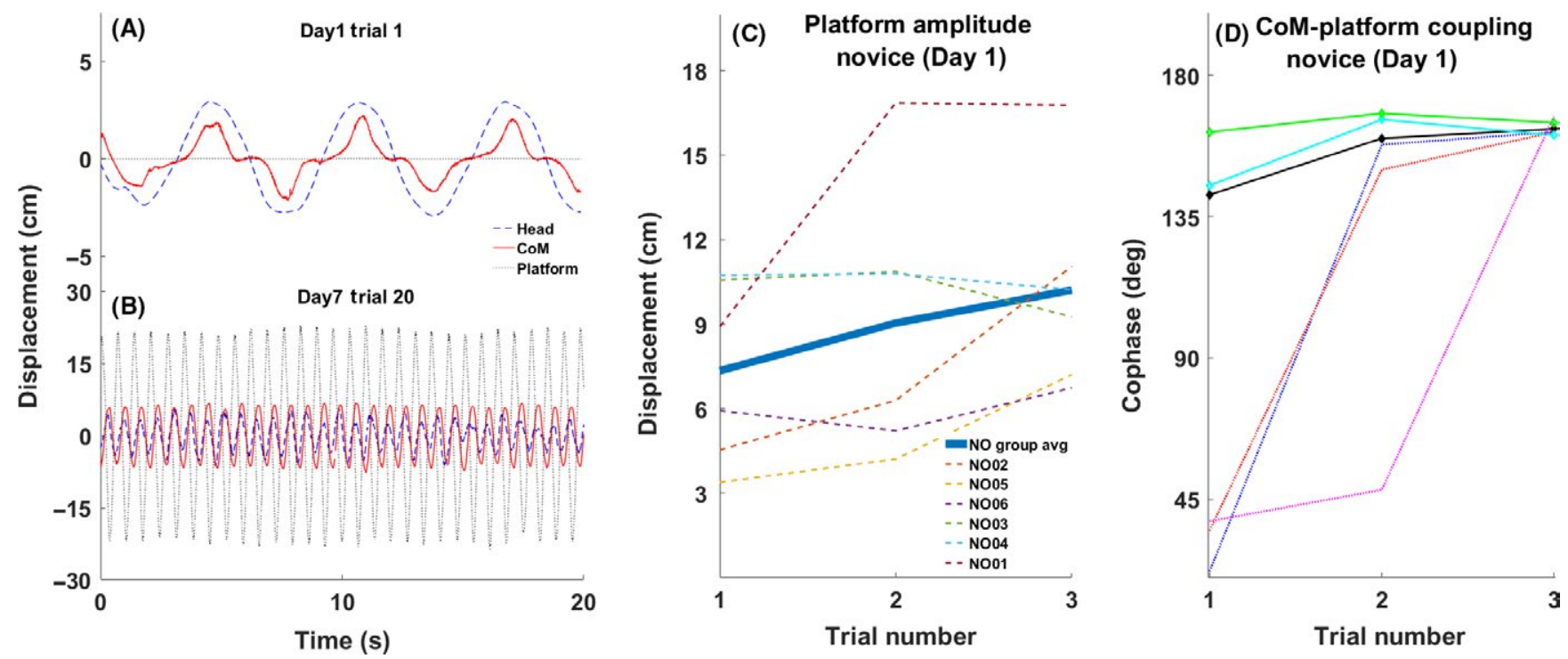

F I G URE 2 Representative novice subject-(A)-day 1, trial 1, (B)-day 7, trial 20, (C)-platform amplitude for novice group and group average for day 1, trials 1-3, (D) cophase values of CoM-platform (ML plane) all novices for day 1, trials 1-3 

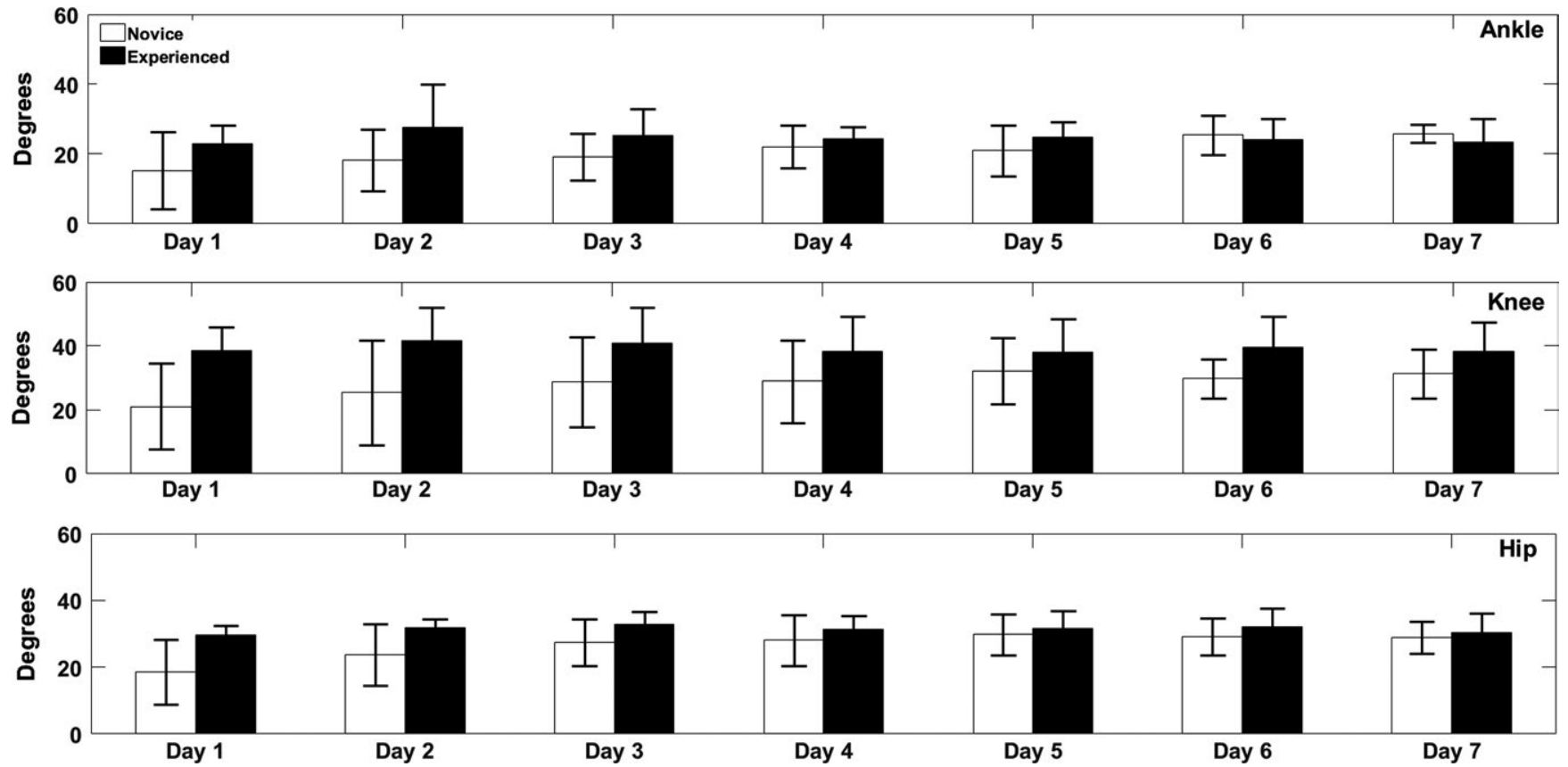

F I G U R E 3 Group mean of joint angle range (ankle, knee, and hip_error bar between subjects' standard deviation) of experienced and novice groups across practice days

TABLE 1 Group mean coefficient of variation $(\mathrm{CV} \%)$ of experienced and novice groups of their joint (ankle, knee, and hip) motions across practice days

\begin{tabular}{|c|c|c|c|c|c|c|}
\hline \multirow[b]{2}{*}{ Practice } & \multicolumn{2}{|c|}{ Ankle Angle (CV \%) } & \multicolumn{2}{|c|}{ Knee Angle (CV \%) } & \multicolumn{2}{|c|}{ Hip Angle (CV \%) } \\
\hline & Experienced & Novice & Experienced & Novice & Experienced & Novice \\
\hline Day 1 & 7 & 30 & 5 & 16 & 4 & 10 \\
\hline Day 2 & 8 & 34 & 6 & 19 & 5 & 13 \\
\hline Day 3 & 7 & 37 & 6 & 22 & 5 & 15 \\
\hline Day 4 & 7 & 45 & 6 & 22 & 5 & 16 \\
\hline Day 5 & 6 & 43 & 6 & 25 & 5 & 17 \\
\hline Day 6 & 7 & 51 & 6 & 23 & 5 & 17 \\
\hline Day 7 & 7 & 53 & 6 & 24 & 5 & 17 \\
\hline
\end{tabular}

angle, $F(1,70)=50, P<.05$, where the experienced group had larger joint angle motions than the novice group. A twoway repeated ANOVA of 7 (days) $\times 2$ (groups-novice and experienced) on the standard deviation of ankle joint angle showed a significant main effect of group, $F(1,70)=234.84$, $P<.05$. For knee joint angle and hip joint angle standard deviation, the main effect of group was also significant, $F(1,70)=102.71, P<.05$ and $F(1,70)=236.57, P<.05$, respectively.

\section{3 | Cophase}

Figure 3D illustrates the cophase values of the CoM-platform coupling for each individual novice participant on day 1 across trials 1 to 3 . Two novice individuals in the study transitioned from in-phase coupling $\left(\sim 0^{\circ}\right)$ to anti-phase coupling $\left(\sim 180^{\circ}\right)$ between trial 1 and trial 2 . One novice individual transitioned from in-phase to anti-phase coupling between trial 1 and trial 3, whereas the remaining novices and all the experienced participants showed anti-phase CoM-platform coupling on the initial trial of day 1.

Figure 4 illustrates the cophase on the different couplings (CoM-platform, head-platform, hip-ankle, hip-knee, and knee-ankle) across practice days for the two groupsexperienced (left panel) and novice (right panel). The CoM-platform cophase values were around $174^{\circ}$ for the experienced (ex) and $161^{\circ}$ for the novice (no). Similarly, the two groups showed a difference in head-platform coupling, $155^{\circ}$ (ex) and $135^{\circ}$ (no), respectively. Regarding the joint motions, the mean cophase values were $77^{\circ}$ (ex) and $76^{\circ}$ (no) for hipankle, $46^{\circ}$ (ex) and $40^{\circ}$ (no) for hip-knee, and $55^{\circ}$ (ex) and $57^{\circ}$ (no) for knee-ankle. 

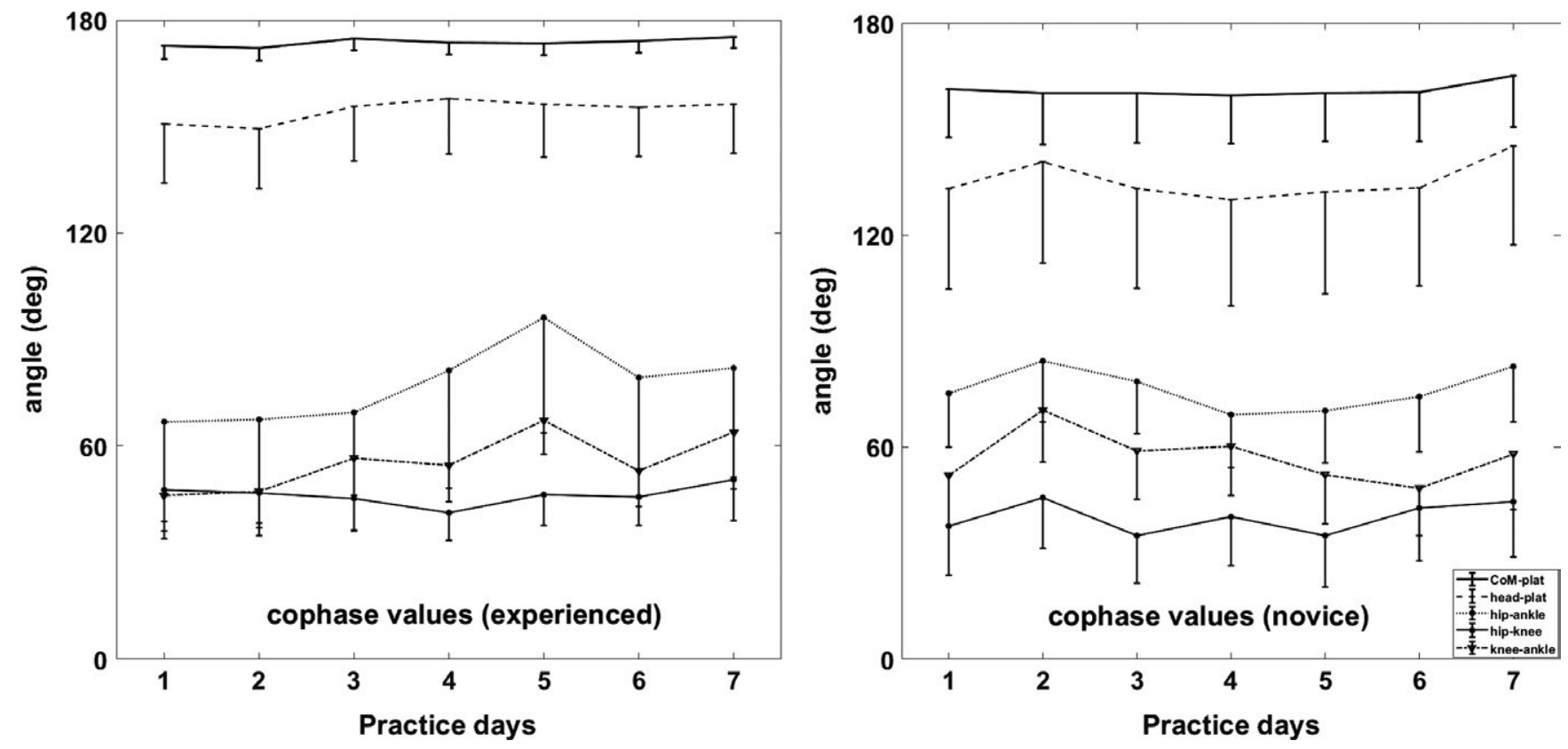

F I G U RE 4 Group mean of cophase of pairwise couplings of experienced and novice groups (error bar between subjects' standard deviation) across practice days
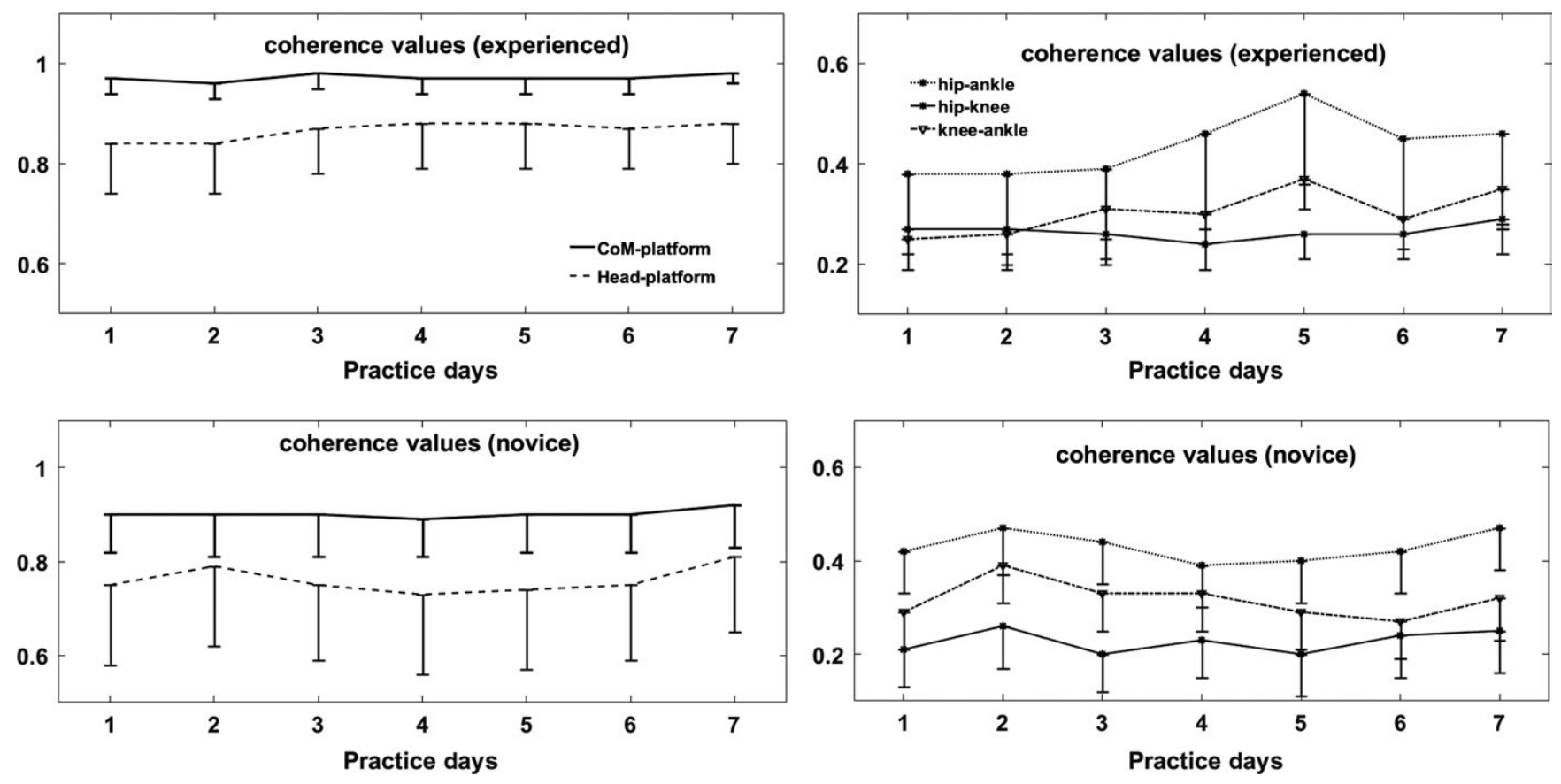

F I G U RE 5 Group mean of coherence of pairwise couplings of experienced and novice groups (error bar between subjects' standard deviation) across practice days

A two-way repeated ANOVA of 7 (days) $\times 2$ (groupsnovice and experienced) on the cophase values of CoMplatform showed a significant main effect of group, $F(1,70)=231.24, P<.05$. Similarly, for head-platform cophase values, the group main effect was significant, $F(1$, $70)=43.69, P<.05$. Although the main effects of days and groups were nonsignificant for the hip-ankle cophase values (dependent variable), there was a significant interaction of group and days, $F(6,70)=2.19, P<.05$. A post hoc Tukey's test showed that for the variable of hip-ankle cophase, the values increased with practice in the experienced but not in the novice group, with groups differing significantly at $P<.05$. For hip-knee cophase values, the main effect of group was significant, $F(1,70)=43.6, P<.05$. 


\section{4 | Coherence}

Figure 5 illustrates the coherence with $S D$ of the different couplings of CoM-platfom, head-platform for experienced (upper left panel), hip-ankle, hip-knee, and knee-ankle for experienced (upper right panel), CoM-platfom, head-platform for novice (lower left panel) and hip-ankle, hip-knee, and knee-ankle for novice (lower right panel). A two-way repeated ANOVA of 7 (days) $\times 2$ (groups-novice and experienced) on the coherence values of CoM-platform showed a significant main effect of group, $F(1,70)=241.26, P<.05$. Similarly, for head-platform coherence values, the group main effect was significant, $F(1,70)=44.37, P<.05$. The interaction of group and days, $F(6,70)=2.23, P=.05$, was significant for hip-ankle coherence. A Tukey-Kramer post hoc showed an interaction of group experience and day 5 , $P=.05$. Tukey's post hoc test showed that coherence of the experienced group was greater than the novice group.

\section{DISCUSSION}

The study investigated the acquisition of a dynamic postural balance task (ski-simulator) as a function of prior practice experience in a related whole-body motor task (downhill skiing). The theoretical and operational focus was to investigate if there were differential qualitative and quantitative pathways of change in the candidate collective variable (CoMplatform), joint synergies, individual joint motions, and task outcome of this multiple $d o f$ task as a function of prior practice experience. We examined if these categories of system variables have differential timescales of change, relations and functional roles of influence in the evolving organization of the dof and task outcome as has been proposed in dynamical systems approaches to motor skill acquisition., ${ }^{1,12,23,33}$

\section{1 | Task outcome}

The platform frequency, amplitude, and velocity were analyzed to investigate the change in task outcome as a function prior practice experience and actual practice on the ski-simulator. ${ }^{3,34,35}$ The two groups clearly showed different pathways of continuous change in task outcome over practice, ${ }^{36,37}$ and this was most evident in progressions of platform amplitude and velocity. The novice group had very limited platform motion on the initial trials of day 1 , particularly in the early trials of the participants showing an in-phase $\mathrm{CoM}$ - platform motion, but they progressively increased the amplitude range of motion and average velocity of the platform over the 7 days.

After the initial trials of day 1 , the platform frequency remained relatively constant (change $<.10 \%$ ) over practice days for both groups, whereas the novice group increased amplitude and average velocity by more than $100 \%$. Indeed, it seems that the learning strategy for both groups once they became "familiarized" or "got the idea" of the task ${ }^{7,38}$ was to increase platform amplitude and average velocity while essentially preserving a modal frequency of the anti-phase CoM-platform motion. Thus, both the phase relation and the frequency of CoM-platform motion are providing an organizational structure of the macroscopic dynamics in this skisimulator task. The relatively stable modal frequency after initial practice is consistent with the proposition that this variable is driving the efficiency of the movement ski-simulator.

We anticipate that further practice would reduce and eventually eliminate the performance difference between the groups. ${ }^{39}$ Nevertheless, the reorganization of the release of the dof by the novice group seems on a slower timescale in this whole-body task than typically is found in the motor learning of upper limb movement scaling tasks. ${ }^{37}$ This difference in the timescales of change in the kinematics reflects the different stages of learning realized by the novice and experienced groups. The slower rate of release of joint angle by the novices is also consistent with the proposal that the stability requirement (staying upright on the simulator) induces a more cautious search strategy in terms of increment of change in the movement kinematics. ${ }^{40}$

The experienced group had practiced downhill skiing for many more hours than were practiced here in the lateral ski motion of the simulator, an experience that clearly induced a positive transfer to both the qualitative and the quantitative movement dynamics of the laboratory ski analogue. An interesting theoretical and open experimental question that has practical ramifications is whether positive transfer also occurs from original practice on the ski-simulator to the actual activity of downhill skiing.

\section{2 | CoM-platform relation}

A central focus was an examination of Bernstein (1967) dof problem in the early stage of skill acquisition (freezing the redundant $d o f$ ) and the subsequent stages of skill acquisition (including freeing the redundant $d o f$ ). In an earlier study, evidence was shown for the phenomena of freezing and freeing $d o f$ as a function of practice in the ski-simulator task. ${ }^{3}$ However, previous ski-simulator and acquisition of multiple dof coordination studies have not compared the pathways of change of individual joint motions and their couplings with the change of a candidate collective variable, together with how prior skill level influences such motor learning. ${ }^{13,16,39,41}$

The findings showed that there were both qualitative (see Figure 2D) and quantitative (see Figures 4 and 5) patterns of change as a function of practice time in learning the lateral skiing movement. Prior practice experience in a related task was found to influence the probability of producing on the initial trial an anti-phase mode to CoM-platform motion. 
All experienced skiers showed an anti-phase mode of CoMplatform on trial 1 that was performed consistently through the practice days, whereas three (50\%) novices showed a phase transition from an in-phase to anti-phase CoM-platform coordination mode between trial 1 and trial 3 on day 1 . However, the three novices that transitioned the CoM-platform relative phase did so after, in effect, minimal practice. The relatively rapid transition with practice is consistent with the position that a relatively stable mode of anti-phase CoM-platform was available that required merely familiarization through preliminary practice in context to induce.

This finding on the rapid change in the qualitative movement dynamics is consistent what Fitts (1964) called "getting the idea" of the task in motor learning. The rapid transition from in-phase to anti-phase for the subset of novice learners holds parallels with the experimental evidence from the HKB model for bimanual control. ${ }^{10}$ In contrast, the learning of the rollerball task that has shown performance discontinuities with differential timescales of change across practice that reflects the freeing of dof at different stages of practice. ${ }^{6}$ It appears that the timescale of forming a macroscopic variable for a coordination pattern in a novel motor task is likely to have considerable variation over participants and tasks.

The findings showed that as a function of practice the inverted pendulum mode of CoM-platform switched to a hanging pendulum mode with progressively large amplitude in the distal end (platform) and constrained amplitude at the pivoted end (head) to reflect the learning of a new anti-phase coordination pattern. ${ }^{3,16}$ Only the anti-phase CoM-platform mode provides the biomechanical support to the freeing of the joint space dof and a larger amplitude and more rapid lateral oscillatory movement pattern. This reveals that the freezing and freeing of the individual joint space motions as articulated by Bernstein (1967) need to be considered in the context of the macroscopic organizing or collective variable for the task. ${ }^{11,12,42}$

We interpret the transition in the CoM-platform coordination pattern to be consistent with the hypothesis of it being the collective variable for this task. The experiment did not test this proposition by scaling a movement property as a control variable as shown originally in the HKB model ${ }^{10}$ of bimanual coordination and for an externally driven platform posture dynamic balance task. ${ }^{11}$ Rather, here we had a selfgenerated motion by learners with different prior practice experience and actual practice acting as a control variable that influenced the formation and expression of the global variable of CoP-platform coupling and the differential scaling of the individual and synergetic joint motions.

The organization of the dof showed also that the CoM and head motions can reflect independent kinematic properties when compared from the first (day 1, trial 1) to the last phase of practice (day 7, trial 20) (see Figure 2A,B). A subset of the novice participants adopted initially in practice an inverted pendulum mode with large amplitude in the distal end (head) and constrained amplitude at the pivoted end (platform) that was essentially in-phase. The CoM motion when compared to head motion as a function of increasing platform velocity reveals that CoM has a slower rate of change and was more stable as a function of the emergent control parameter (platform velocity). Thus, our findings show that head motion can become independent to some degree of the motion of the CoM given the confluence of constraints to movement in action ${ }^{43}$ including the task demands. ${ }^{44}$

\section{3 | Joint motion excursions and couplings}

Prior practice experience significantly facilitated the release with practice of the excursion of motion at the individual joint (ankle, knee, and hip) dof but this only took place within the anti-phase CoM-platform mode. Thus, release of the individual dof and the increased excursion of joint motion were strongly influenced by prior practice experience and the adoption of the anti-phase CoM-platform mode. We postulate that the relatively slower rate of change (release) in joint motion by novice learners over practice was due to the stability demands of the dynamic postural task and a conservative approach to the perceived negative consequences of the loss of balance on the ski-simulator.

The coherence values of the coupling of CoM, head, platform, hip, knee, and ankle motion along ML direction revealed that the experienced group was already attuned to the demands of the ski-simulator task and hence executed antiphase coupling of CoM-platform from trial 1, day 1 unlike $50 \%$ of the novice group. The coherence analysis showed that the coordination dynamics of the CoM-platform coupling had distinct coherence values $(\sim 1)$ when compared to the coupled synergy variables $(\sim<0.5)$ across practice days (see Figure 5) for both groups. The intermediate coherence values of synergy variables (hip-knee-ankle pairings) provide further evidence that the coupling of the synergies was on a different timescale from that of the candidate collective variable-a feature that can emerge in the multiple dof task. ${ }^{11}$ Overall, for both groups, the coupling measures of coherence and cophase of the joint motions showed little persistent change over the 7 days of practice and little influence of prior practice experience, consistent with a reflection of a different functional role than that of the candidate collective variable and the regulation of the task outcome. Indeed, where a drift in mean value of the cophase or coherence for the synergies occurred the relative level of change was considerably smaller than the order of magnitude changes in the task outcome, individual joint motions, and the CoM-platform relative phase (for those participants who showed the transition).

The multiple patterns of change in the different categories of variables over practice time reflect the flexibility and degeneracy of the system and the challenge of understanding 
change and learning in a nonlinear system with multiple $d o f .{ }^{1}$ Nevertheless, the findings revealed continuity in the incremental progression of task outcome (platform position and velocity) in both the novice and the experienced groups in a way that is consistent with traditional findings of change in task outcome through motor learning. ${ }^{36,37}$ This does not mean that the change in task outcome in motor learning is always continuous and progressive ${ }^{6}$ or that the persistent change over practice time in the collective variable, individual joint motions, or synergies will follow the differential patterns and timescales shown here.

\section{4 | Perspectives}

The findings show that in learning this whole-body sportsrelated ski-simulator task prior experience and practice induce different rates of change in the categories of movement variables, including task outcome, the candidate collective variable, neuromuscular synergies, and joint motions. Traditional emphasis in motor skill learning has been on achieving the task outcome and to a lesser extent the role of the change in joint motions to realize this goal. Here, however, the functional role of the macroscopic variable of CoM-platform motion (phase relation and frequency) is revealed in organizing the motions of the individual dof and joint motions. The release of the individual joint dof with practice ${ }^{16}$ is dependent on the global organization of the movement system. The formation of the macroscopic movement dynamics through practice is an under-researched problem of the early stage of skill learning previously described as getting the idea of the task. ${ }^{7,38}$ The findings show the functional role of the integration of postural and limb motion dynamics in a sports-related task and provide a new direction of inquiry for the many existing instructional strategies of motor skill acquisition. ${ }^{45}$

\section{ACKNOWLEDGEMENTS}

The authors are grateful to Tim Benner for technical support.

\section{CONFLICT OF INTEREST}

Aviroop Dutt-Mazumder and Karl Newell declare that they have no conflict of interest.

\section{ORCID}

A. Dutt-Mazumder (iD) http://orcid. org/0000-0002-6256-0578

\section{REFERENCES}

1. Bernstein NA. The Coordination and Regulation of Movements. Oxford, UK: Pergamon Press Ltd; 1967.
2. Arutyunyan GA, Gurfinkel VS, Mirskii ML. Study of taking aim at a target. Biophysics (Oxf). 1968;13:642-645.

3. Vereijken B, van Emmerik REA, Whiting HTA, Newell KM. Free (z) ing Degrees of freedom in skill acquisition. J Mot Behav 1992;24:133-142.

4. Winter DA. Biomechanics and Motor Control of Human Movement. John Wiley \& Sons, NJ: John Wiley \& Sons, Ltd.; 2009.

5. Dutt-Mazumder A, Challis JH, Newell KM. Maintenance of postural stability as a function of tilted base of support. Hum Mov Sci. 2016;48:91-101.

6. Liu Y-T, Newell KM. S-Shaped motor learning and nonequilibrium phase transitions. J Exp Psychol Hum Percept Perform. 2015;41:403-414.

7. Fitts PM. Perceptual-motor skill learning. In: Melton AW, ed. Categories of Human Learning. London, UK: Academic Press; 1964:243-285.

8. Mitra S, Amazeen PG, Turvey MT. Intermediate motor learning as decreasing active (dynamical) degrees of freedom. Hum Mov Sci. 1998;17:17-65.

9. Dutt-Mazumder A, Slobounov SM, Challis JH, Newell KM. Postural stability margins as a function of support surface slopes. PLOS ONE. 2016;11:e0164913.

10. Haken H, Kelso JAS, Bunz H. A theoretical model of phase transitions in human hand movements. Biol Cybern. 1985;51:347-356.

11. Ko J-H, Challis JH, Newell KM. Transition of COM-COP relative phase in a dynamic balance task. Hum Mov Sci. 2014;38:1-14.

12. Kelso JAS. Dynamic Patterns: The Self-Organization of Brain and Behavior. Cambridge, UK: MIT Press; 1995.

13. Hong SL, Newell KM. Practice effects on local and global dynamics of the ski-simulator task. Exp Brain Res. 2006;169:350-360.

14. Hong SL, Newell KM. Change in the organization of degrees with learning. J Mot Behav. 2006;38:88-100.

15. Jeka J, Kelso JAS. The dynamic pattern approach to coordinated behavior: a tutorial review. In: Wallace SA, ed. Perspectives on the Coordination of Movement. Advances in Psychology. NorthHolland: Elsevier Science Publishers 1989:3-46.

16. Vereijken B, van Emmerik REA, Bongaardt R, Beek WJ, Newell KM. Changing coordinative structures in complex skill acquisition. Hum Mov Sci. 1997;16:823-844.

17. Whiting HTA, Bijlard MJ, den Brinker BPLM. The effect of the availability of a dynamic model on the acquisition of a complex cyclical action. Q J Exp Psychol Sect A. 1987;39:43-59.

18. Kostrubiec V, Tallet J, Zanone P-G. How a new behavioral pattern is stabilized with learning determines its persistence and flexibility in memory. Exp Brain Res. 2006;170:238-244.

19. Kostrubiec V, Zanone P-G, Fuchs A, Kelso JAS. Beyond the blank slate: routes to learning new coordination patterns depend on the intrinsic dynamics of the learner-experimental evidence and theoretical model. Front Hum Neurosci. 2012;6:222.

20. Chow JY, Davids K, Button C, Rein R. Dynamics of Movement Patterning in Learning a Discrete Multiarticular Action. Mot Control. 2008;12:219-240.

21. Chow JY, Davids K, Button C, Koh M. Variation in coordination of a discrete multiarticular action as a function of skill level. J Mot Behav. 2007;39:463-479.

22. Chow J, Davids K, Button C, Koh M. Coordination changes in a discrete multi-articular action as a function of practice. Acta Psychol (Amst). 2008;127:163-176. 
23. Kugler PN, Kelso JAS, Turvey MT. On the concept of coordinative structures as dissipative structures: I. Theoretical lines of convergence. Tutorials Mot Behav. 1980;3:3-47.

24. Newell KM. Constraints on the development of coordination. In: Whiting HTA, Wade MG, eds. Motor Development in Children: aspects of Coordination and Control. Dordrecht, the Netherlands: Martinus Nijhoff Publishers; 1985:341-360.

25. Newell KM, Liu YT, Mayer-Kress G. Time scales in motor learning and development. Psychol Rev 2001;108:57-82.

26. Savelsbergh GJ, Maas HLJ, Geert P. Non-Linear Developmental Processes. Amsterdam, the Netherlands: Royal Netherlands Academy of Arts and Sciences; 1999.

27. Dempster. Space Requirements of the Seated Operator. (WADC Technical report,55-159). Ohio: Aerospace Medical Research Laboratory; 1955.

28. Creath R, Kiemel T, Horak F, Peterka R, Jeka J. A unified view of quiet and perturbed stance: simultaneous co-existing excitable modes. Neurosci Lett. 2005;377:75-80.

29. Bloomfield P. Fourier Analysis of Time Series: an Introduction. New York, NY: John Wiley \& Sons; 2004.

30. Mardia KV. Statistics of directional data. J R Stat Soc Ser B. 1975;37:349-393.

31. Mitra P, Bokil H. Observed Brain Dynamics. New York, NY: Oxford University Press; 2008.

32. Thompson D. Spectrum estimation and harmonic analysis. Proc IEEE. 1982;70:1055-1096.

33. Newell KM, Vaillancourt DE. Dimensional change in motor learning. Hum Mov Sci. 2001;20:695-715.

34. den Brinker BPLM, van Hekken MF. The analysis of slalom-ski type movements using a ski-simulator apparatus. Hum Mov Sci 1982;2:91-108.

35. Wulf G, Höß M, Prinz W. Instructions for motor learning: differential effects of internal versus external focus of attention. $J$ Mot Behav. 1998;30:169-179.

36. Newell A, Rosenbloom PS. Mechanisms of skill acquisition and the law of practice. Cogn Ski their Acquis. 1981;1:1-55.
37. Schmidt RA, Lee TD. Motor Learning and Performance, $5 E$ with Web Study Guide: From Principles to Application. Champaign, IL: Human Kinetics; 2013.

38. Gentile AM. A Working Model of Skill Acquisition with Application to Teaching. Quest. 1972;17:3-23.

39. Nourrit D, Delignières D, Caillou N, Deschamps T, Lauriot B. On discontinuities in motor learning: a longitudinal study of complex skill acquisition on a ski-simulator. J Mot Behav. 2003;35:151-170.

40. Orth D, Davids K, Seifert L. Constraints representing a metastable régime facilitate exploration during practice and transfer of learning in a complex multi-articular task. Hum Mov Sci 2017;57:291-302 .

41. Wulf $\mathrm{G}$, Weigelt $\mathrm{C}$. Instructions about physical principles in learning a complex motor skill: to tell or not to tell... Res Quaterly Exerc Sport. 1997;68:362-367.

42. Dutt-Mazumder A, Newell KM. Transitions of postural coordination as a function of frequency of the moving support platform. Hum Mov Sci. 2017;52:24-35.

43. Horak FB, Shupert CL, Dietz V, Horstmann G. Vestibular and somatosensory contributions to responses to head and body displacements in stance. Exp Brain Res. 1994;100:93-106.

44. Bardy BG, Oullier O, Bootsma RJ, Stoffregen TA. Dynamics of human postural transitions. J Exp Psychol Hum Percept Perform. 2002;28:499-514.

45. Davids K, Button C, Bennett S. Dynamics of Skill Acquisition: A Constraints-Led Approach. Champaign, IL: Human Kinetics; 2008 .

How to cite this article: Dutt-Mazumder A, Newell KM. Task experience influences coordinative structures and performance variables in learning a slalom ski-simulator task. Scand J Med Sci Sports. 2018;28:1604-1614. https://doi.org/10.1111/sms.13063 